\section{Encuesta sobre el ejercicio en niños residentes de Estados Unidos}

La tendencia a llevar una vida sedentaria se observa no solo en la población adulta, sino también en los niños, según una encuesta telefónica realizada en 2002 en Estados Unidos de América en niños de 9 a 13 años de edad y sus padres. La encuesta, que abarcó una muestra aleatoria de alrededor de 3600 hogares en todo el país, reveló que $61,5 \%$ de los niños de 9 a 13 años de edad no practican ningún deporte ni ejercicio organizado en sus horas libres y que durante estas horas $22,6 \%$ no participan en ningún tipo de actividad física. Se torna necesario, dadas las implicaciones del sedentarismo para la salud (hipertensión arterial, gordura, trastornos cardiovasculares, problemas psicosociales, etc.), adoptar medidas para incentivar a las personas jóvenes a hacer más ejercicio.

Para fines del estudio el ejercicio organizado se definió como la participación en algún tipo de ejercicio en grupo bajo la dirección de un entrenador durante los 7 días anteriores a la encuesta. La actividad física en horas libres se definió como la participación en alguna actividad física fuera del ámbito escolar. A los padres se les pidió que opinaran acerca de cinco posibles barreras a la participación de sus hijos en actividades físicas: problemas de transporte, falta de oportunidades para participar en actividades físicas cerca de la casa, carestía, falta de tiempo debido a las obligaciones de los padres y preocupación por la situación de seguridad en el vecindario.

La encuesta reveló que la participación en actividades físicas organizadas es menor en grado estadísticamente significativo entre niños hispanos, niños de raza negra sin ascendencia hispana, y niños cuyos padres tienen pocos ingresos y poca escolaridad. En general todos los padres coincidieron en percibir las mismas barreras al ejercicio físico, pero los padres de niños hispanos y de raza negra sin ascendencia hispana adujeron como barreras los problemas de transporte, la carestía y la falta de oportunidades con una frecuencia significativamente mayor que los padres de niños blancos sin ascendencia hispana. La preocupación por la seguridad en el vecindario fue expresada con mayor frecuencia en conexión con las niñas que con los niños y por los padres de niños hispanos que por los de niños blancos o negros no hispanos. En términos generales, los padres con menores ingresos y menor escolaridad mencionaron más barreras que otros padres.

Las actividades físicas organizadas practicadas con mayor frecuencia, independientemente de la raza, edad o sexo, fueron el béisbol, el baloncesto y el fútbol. Las actividades físicas practicadas con mayor frecuencia en horas libres fueron, en primer lugar, montar en bicicleta y jugar al baloncesto, seguidos de caminar, correr y participar en juegos que exigen movimiento físico.

Es primera vez que se dispone de información de alcance nacional en Estados Unidos acerca del grado y tipo de actividad física en que participan los niños de 9 a 13 años. La conclusión es que estos niños en su mayoría hacen algún tipo de ejercicio en su tiempo libre, pero que es necesario adoptar medidas para lograr que más niños hagan el ejercicio que deben. Si se considera que los hábitos de vida se adquieren en la infancia, cualquier inversión en incrementar la actividad física de los niños redundará en una futura población adulta más activa. (Duke J, et al. Physical activity levels among children aged 9-13 years - United States, 2002. MMWR 2003;52(33):785-788).

\section{Conexión entre la obesidad crónica en niños y la conducta rebelde}

Según los resultados de un estudio de 8 años de duración realizado por investigadores de la Universidad de Duke en Carolina del Norte, Estados Unidos de América, los niños que tienen obesidad crónica son más propensos a sufrir ciertos trastornos mentales que otros niños. El estudio, que se basó en una muestra de casi 1000 niños de 9 a 16 años de edad, reveló que entre los que se mostraron obsesos en cada uno de sus seguimientos anuales hubo una mayor frecuencia de conductas de tipo rebelde, independientemente del sexo, y que los varones con obesidad crónica mostraron una mayor tendencia a tener síntomas de depresión. Estos riesgos no se detectaron, sin embargo, en niños cuyo peso fluctuó de un año para otro ni en aquellos cuya obesidad empezó durante la niñez tardía o la adolescencia. Ya se conocía la asociación entre la obesidad en la niñez y adolescencia y toda una serie de problemas de salud -afecciones respiratorias, digestivas y circulatorias en la propia niñez, así 
como trastornos cardiovasculares, diabetes y cáncer en la edad adulta-, pero estos hallazgos apuntan a una asociación entre la obesidad en edad temprana y algunos trastornos psiquiátricos en niños.

Los niños y adolescentes estudiados por el grupo de investigadores de la Universidad de Duke participaban en un estudio de larga duración sobre trastornos mentales. En él se halló, además de lo descrito anteriormente, que la frecuencia de la obesidad aumenta con la edad, habiéndose encontrado obesidad solamente en la niñez tardía en 5\% de los casos y en la adolescencia solamente en 7,5\%. Se observó una frecuencia de obesidad crónica de 15\% para sorpresa de los investigadores, quienes pronosticaron una frecuencia tres veces más baja basándose en los criterios más recientes del Centro para el Control y la Prevención de Enfermedades.

En términos conductuales, la rebeldía fue dos veces más frecuente en niños obesos que en niños sin obesidad, independientemente del sexo, y la depresión se observó con una frecuencia cuatro veces mayor en varones obesos que en varones sin obesidad. En cambio, en niñas no se detectó ninguna asociación entre la obesidad y la depresión. Se desconocen los motivos de estas asociaciones. No obstante, es posible que el repudio y la burla de sus compañeros predispongan a los niños obesos a la depresión y a problemas del comportamiento, aunque tampoco se descarta lo contrario, es decir, que los trastornos psiquiátricos predispongan a los niños a la obesidad. Sea como fuere, los padres harían bien en enseñarles a sus hijos las reglas de la buena alimentación, procurando a la vez inculcarles que la obesidad es mala para la salud y evitando reforzar cualquier complejo que puedan tener como resultado de su obesidad. (Mustillo S, et al. Obesity and psychiatric disorder: developmental trajectories. Pediatrics 2003;111:851-859).

\section{Caminar mejora la tolerancia a la glucosa en mujeres con sobrepeso}

Se ha comprobado que el ejercicio físico es beneficioso para las personas con diabetes tipo 2, en quienes ayuda a reducir la glucemia, aumentar la sensibilidad a la insulina y reducir la obesidad y el riesgo de trastornos cardiovasculares. Lamentablemente, las exigencias de la vida laboral interfieren con la práctica de algún tipo de actividad física estructurada y fomentan un estilo de vida sedentario, aun cuando los beneficios del ejercicio se hacen cada vez más patentes. Según los datos arrojados por un estudio del Jefe de Sanidad de los Estados Unidos de América, muy pocos estadounidenses practican un régimen estructurado de ejercicios. Como resultado, el Colegio Estadounidense de Me- dicina Deportiva (CEMD) y los Centros para el Control y la Prevención de Enfermedades (CDC) en Atlanta, Georgia, recomiendan que toda persona adulta acumule por lo menos 30 minutos de ejercicio moderadamente intenso todos los días. La recomendación es fácil de cumplir con solo caminar alrededor de tres kilómetros a paso acelerado todos los días. Una persona sedentaria normalmente camina alrededor de 4000 a 6000 pasos al día, de tal manera que con solo caminar tres kilómetros adicionales (unos 4000 pasos) alcanzará los 10000 pasos diarios, que es el número recomendado en la actualidad. La recomendación de los 10000 pasos tiene ventajas: es fácil de observar y el ejercicio tiene carácter acumulativo, lo cual facilita su incorporación a la rutina diaria. Por otra parte, hasta la fecha no se ha determinado si su cumplimiento redunda en beneficios sanitarios específicos.

Investigadores de la Universidad de Tennessee en Knoxville emprendieron un estudio de 12 semanas con 18 mujeres voluntarias de 40 a 65 años de edad. El objeto era determinar si caminar 10000 pasos al día durante 8 semanas era suficiente para mejorar la tolerancia a la glucosa en mujeres sedentarias y con sobrepeso que estaban en riesgo de sufrir diabetes tipo 2. Todas las participantes tenían antecedentes familiares de este tipo de diabetes y un índice de masa corporal (IMC) $>25,0 \mathrm{~kg} / \mathrm{m}^{2}$ sostenido durante un mínimo de 6 meses. Diez de las mujeres eran posmenopáusicas y cinco de ellas recibían terapia de restitución hormonal. Tres mujeres tenían, además, antecedentes de diabetes gestacional y 11 tenían un diagnóstico de hipertensión arterial. El estudio se diseño de tal manera que las propias mujeres sirvieron de pacientes y testigos simultáneamente. Durante las primeras 4 semanas se les pidió que no alteraran su actividad física habitual; en las 8 semanas posteriores las mujeres fueron sometidas a una intervención que consistió en hacerlas caminar 10000 pasos diarios en total. Los pasos se contaron mediante un podómetro y todo ejercicio fue anotado en un registro de actividades. A las mujeres se les prohibió que se pusieran a régimen dietético durante el estudio.

Al final del estudio, el valor de glucosa en la sangre a las 2 horas de ingerida la carga de glucosa oral había disminuido un $11 \%(P<0,001)$ en promedio. No se encontraron diferencias significativas entre pacientes y testigos en los valores de glucosa en sangre por debajo de la curva (VDC), la glucosa plasmática en ayunas, la glucosa plasmática máxima, la insulina en ayunas y la insulina a las 2 horas de ingerida la carga oral de glucosa. No obstante, la presión arterial sistólica al final del estudio fue más baja $(P=0,001)$ que a su inicio y a las 4 semanas, $\mathrm{y}$ la diastólica fue más baja tanto a las cuatro semanas $(P=0,002)$ como al final del estudio $(P=0,014)$. No 
se observaron cambios significativos en el porcentaje de grasa corporal, la circumferencia de la cintura, o la relación entre la cintura y la cadera. Tampoco se observó ninguna diferencia significativa en el IMC.

En resumen, el estudio reveló que el ejercicio practicado en forma de 10000 pasos diarios, que es lo recomendado por el CEMD y el CDC, mejora la tolerancia a la glucosa y reduce notablemente la presión arterial sistólica y diastólica. (Swartz AM, et al. Increasing daily walking improves glucose tolerance in overweight women. Prev Med 2003; 37(4):356-362).

\section{¿Puede el ejercicio en la edad madura prevenir o reducir la discapacidad?}

Muchas personas de edad avanzada con discapacidades o limitaciones funcionales cifran sus esperanzas en el ejercicio para obtener alivio o prevenir la progresión de los síntomas. Si bien es cierto que los beneficios de la actividad física moderada o intensa para prevenir o mejorar las limitaciones funcionales han sido confirmados en numerosos estudios, la situación en el caso de las discapacidades es menos clara debido a que los estudios efectuados hasta el momento han arrojado datos contradictorios. Esto es, al menos, lo que reveló una reciente revisión bibliográfica efectuada por un investigador de la Universidad de Boston, Massachusetts. En lo que respecta a la eficacia del ejercicio para reducir o prevenir la discapacidad, los estudios prospectivos han sido más alentadores que los experimentales, que en muchos casos no han mostrado mejoramiento alguno. Nada de esto significa que el ejercicio no tenga utilidad. Obviamente, estas discrepancias en los resultados de los estudios reflejan en cierta medida diferencias en la definición de las variables dependientes e independientes: "discapacidad", "limitación funcional", "actividad física" y "ejercicio". La situación la viene a complicar el hecho de que en personas de edad avanzada ciertas actividades pueden interpretarse como "actividad física" en algunos casos y como "ejercicio" en otros. Normalmente, "actividad física" se define como "cualquier movimiento corporal con contracción del músculo esquelético que hace que el gasto energético supere perceptiblemente el del nivel basal", y "ejercicio" se define como "movimientos corporales planificados, estructurados y repetitivos que se realizan con o sin fines de acondicionamiento físico".

El investigador hizo una revisión de todos los estudios publicados en inglés entre 1995 y 2002 sobre el efecto de la actividad física y el ejercicio en personas de 60 años de edad o mayores con las limitaciones funcionales normales de su edad - dificultad para caminar ligero o para subir y bajar escaleras, por ejemplo- o con discapacidades oca- sionadas por trastornos musculoesqueléticos o de otro tipo. Sobre la base de los estudios examinados cabe afirmar que caminar y hacer ejercicios aerobios y de fortalecimiento mejoran la agilidad de personas ancianas para caminar y, en algunos casos, para subir y bajar escaleras y pararse y sentarse, aun en presencia de osteoartritis de rodillas o caderas. La actividad física también parece proteger contra las limitaciones funcionales de la vejez. Aunque los resultados de estudios controlados aleatorizados en personas mayores con discapacidades fueron más contradictorios, muchos de ellos mostraron un beneficio. Se desprende claramente de la revisión efectuada que el ejercicio en la edad avanzada mejora la fuerza muscular, la capacidad aerobia y la agilidad, particularmente para caminar. Los estudios prospectivos también apuntan a una relación inversa entre el ejercicio y la discapacidad. (Keysor JJ. Does late-life physical activity or exercise prevent or minimize disablement? A critical review of the scientific evidence. 2003;25(Supl 2):129-136).

\section{La actividad física y su efecto exclusivo sobre el riesgo de obesidad y diabetes}

Diversos estudios de carácter observacional y experimental han demostrado que la actividad física, cuando se combina con una dieta adecuada y pérdida de peso, se asocia con un riesgo reducido de diabetes tipo 2. Intervenciones de este tipo a gran escala en personas de distintas razas y extracciones étnicas con tolerancia reducida a la glucosa han dado por resultado una menor incidencia de diabetes tipo 2 en varios países, entre ellos China, Finlandia y Estados Unidos. No obstante, el efecto independiente de la actividad física nunca se había puesto a prueba directamente hasta hace poco, cuando un grupo de investigadores estadounidenses partieron de la hipótesis de que la actividad física protege contra la diabetes tipo 2 , independientemente de otros factores. Su hipótesis se basó en los resultados de estudios anteriores según los cuales la actividad física mejora la sensibilidad a la insulina, al margen de su efecto sobre la pérdida de peso y la distribución de la grasa corporal. Asimismo, en un estudio de corte transversal se demostró una relación inversa entre la actividad física y la concentración de insulina en dos poblaciones en alto riesgo de sufrir diabetes que diferían radicalmente en sus respectivos índices de masa corporal (IMC). Esta relación se ha visto confirmada también en poblaciones indígenas, de tal manera que todo apunta a que la actividad física ejerce un efecto benéfico por incremento de la sensibilidad a la insulina que no guarda relación con su efecto sobre el peso ni la composición corporal. 
Los indios pimas de Arizona, Estados Unidos, tienen una de las incidencias más altas de diabetes tipo 2 en el mundo. Por tal motivo los autores del estudio estadounidense ya mencionado eligieron a esta población para investigar el efecto independiente de la actividad física. Su muestra se compuso de 1728 indígenas entre los 15 y 59 años de edad, a quienes se les administró un cuestionario para documentar su actividad física en horas libres $\mathrm{y}$ en el trabajo. Estas personas además tuvieron un seguimiento de 6 años, período durante el cual 346 se hicieron diabéticas, de acuerdo con los resultados de pruebas de tolerancia a la glucosa administrada por la vía oral. Al final del estudio, se encontró que la actividad física total estaba relacionada con la incidencia de diabetes en mujeres y en hombres, aunque solamente en mujeres se observó una relación estadísticamente significativa $(P<0,05)$. Después de hacer ajustes en función del IMC, la relación entre la actividad física y la incidencia de diabetes se debilitó en personas de uno y otro sexo. Cuando estas tasas de incidencia ajustadas se examinaron a la luz de los niveles de actividad física estratificados por tercios de IMC, la tasa de incidencia siguió siendo más baja en hombres y mujeres con niveles de actividad más altos, sin importar su IMC. La única excepción en este sentido fue la del grupo de hombres en el tercio mediano en cuanto a IMC. En su conjunto, estos resultados indican que la actividad física, cuando se practica con regularidad como parte del estilo de vida, puede desempeñar un papel importante en la prevención de la diabetes tipo 2, incluso en poblaciones en riesgo de sufrir la enfermedad. (Kriska AM, et al. Physical activity, obesity, and the incidence of type 2 diabetes in a high-risk population. Am J Epidemiol 2003;158:669-675.)

\section{¿Puede la actividad física reducir el riesgo de accidentes cerebrovasculares?}

Los accidentes cerebrovasculares son una de las causas de muerte más importantes en países desarrollados y cada vez más en países en desarrollo como resultado de la transición epidemiológica y el envejecimiento de la población. Además, de las personas que sobreviven, muchas quedan discapacitadas y requieren rehabilitación y atención médica prolongada, con un enorme costo para los servicios de salud y la sociedad en general. Los principales factores que predisponen a los accidentes cerebrovasculares son la hipertensión arterial y la enfermedad coronaria, y ambos pueden reducirse mediante un régimen de actividad física regular. Pero además, hay posibilidades de que la actividad física tenga también un efecto protector independiente contra los accidentes cerebrovasculares, ya documentada en algunos estudios, aunque otros no han arrojado ninguna asociación o hasta han mostrado una asociación directa entre el nivel de actividad y la incidencia de accidentes cerebrovasculares o la mortalidad por esta causa.

De lo anterior deriva la importancia de un estudio reciente realizado por investigadores en Texas, Estados Unidos, quienes examinaron la posibilidad de una asociación entre la actividad física y la incidencia de accidentes cerebrovasculares o la mortalidad por dichos accidentes en estudios publicados entre 1996 y 2002 e indizados en MEDLINE, más el informe del Jefe de Sanidad de Estados Unidos, publicado en 1996, sobre la actividad física y la salud. Las palabras de búsqueda fueron physical activity, exercise, leisure-time activity, stroke, y cardiovascular disease. En total se identificaron 23 estudios epidemiológicos -18 estudios de cohorte y 5 de casos y testigos- que satisfacían los criterios de inclusión.

Este metaanálisis dio por resultado una demostrada reducción del riesgo de accidentes cerebrovasculares de todo tipo - hemorrágicos y embólicos- en personas que llevaban una vida con un nivel de actividad física moderado o intenso o que estaban en buena forma física, por oposición a las que tenían poca actividad física o se encontraban en mala forma física. Esta asociación se observó tanto en los estudios de cohorte como en los de casos y testigos y ambos tipos combinados. En los estudios de cohorte se detectó una disminución de $25 \%$ del riesgo de sufrir un accidente cerebrovascular o de morir por esta causa en personas con una vida de intensa actividad física ( $R R=0,75$; IC95\%: 0,69 a 0,82 ) en comparación con personas poco activas. En lo referente a estudios de casos y testigos, las personas muy activas tuvieron un riesgo de sufrir accidentes cerebrovasculares o de morir de ellos $64 \%$ más bajo que las poco activas ( $R R=0,36$; IC $95 \%$ : $0,25$ a 0,52$)$. Cuando se combinaron los estudios de cohorte con los de casos y testigos se encontró un riesgo $27 \%$ menor de sufrir accidentes cerebrovasculares o de morir de ellos en las personas más activas por comparación con las menos activas $(\mathrm{RR}=$ 0,73; IC95\%: 0,67 a 0,79). Se puede concluir que la actividad física altamente o moderadamente intensa podría estar asociada con un menor riesgo de todo tipo de accidentes cerebrovasculares, aunque el estudio aquí descrito adoleció de algunas limitaciones, entre ellas la poca precisión de los cuestionarios usados en algunos estudios para determinar el grado de actividad física, la escasez de estudios sobre la relación de la actividad física y los accidentes cerebrovasculares de tipo hemorrágico, y variaciones en la definición de actividad intensa, moderada y leve. (Do Lee, et al. Physical activity and stroke risk. Stroke 2003;34:2475-2486.) 